\section{La malaria en las Américas y las actividades de control}

En 1995, 32\% de la población de las Américas vivía en zonas donde las condiciones ecológicas favorecían la transmisión de la malaria. A fin de obtener una idea más precisa de la distribución de la enfermedad, los países de la Región establecieron un sistema de estratificación de zonas maláricas según el riesgo de exposición (alto, mediano o bajo). Este último depende de numerosos factores, entre ellos los movimientos migratorios, la estabilidad social y la frecuencia del contacto entre el ser humano y el vector.

De 1994 a 1995, la incidencia de malaria en las Américas aumentó en 14,6\% debido, en parte, al incremento de las infecciones causadas por Plasmodium falciparum en Bolivia, Colombia y Perú. También se produjo un aumento notable de la tasa de morbilidad en todas las Américas, especialmente en zonas de transmisión activa. En 1995 el Brasil y los países andinos tuvieron el mayor número de casos de malaria en toda la Región (565 727 y 461 121, respectivamente), aunque el mayor riesgo de transmisión se observó en la zona constituida por Guayana Francesa, Guyana y Suriname. La transmisión se intensificó en mayor grado en Belice, Bolivia, Colombia, Nicaragua y Perú.

En zonas de riesgo alto y mediano, la vigilancia epidemiológica en 1995 fue más activa que en 1994. Los últimos años se han caracterizado, además, por una integración de las actividades de búsqueda, diagnóstico y tratamiento inmediato de casos. La vigilancia activa, cuyos costos de operación son altos, ha tenido una eficiencia diagnóstica más baja $(2,42 \%)$ que los servicios de salud locales $(16,9 \%)$. Como resultado de la introducción del sistema de estratificación por zonas de riesgo en los programas nacionales de control, las iniciativas se han concentrado en zonas de alta prioridad. Esta medida y la aplicación sostenida de la "Estrategia mundial para el control de la malaria", con su énfasis en el manejo clínico de la enfermedad, han llevado a un aumento progresivo de la disponibilidad del tratamiento, que actualmente es de 61,5 tratamientos por cada 1000 enfermos.

En los países se siguen llevando a cabo actividades para el control del vector con el fin de prevenir la transmisión. Los plaguicidas organoclorados y organofosforados, tales como el diclorodifeniltri- cloroetano (DDT), han sido reemplazados paulatinamente por los piretroides sintéticos, como el malatión y el fenitrotión. En términos generales, los programas de control enfrentan grandes restricciones presupuestarias y se ven obligados a depender cada vez más de préstamos y subsidios procedentes de fuentes ajenas al sector de la salud. (Pan American Health Organization. Malaria in the Americas. Epidemiol Bull 1996;17:1-11).

\section{La visualización del cerebro en el estudio de la drogadicción}

Según el director del Instituto Nacional del Abuso de Drogas, Estados Unidos de América, los resultados de algunas investigaciones recientes están ayudando a los científicos a entender mejor los complejos procesos cerebrales y conductuales que entran en juego en la drogadicción. Estas nuevas técnicas permiten ver lo que sucede dentro del cerebro humano mientras el individuo recuerda o experimenta los efectos de un narcótico. Antes de la aparición de estos procedimientos, la visualización del cerebro se hacía mediante técnicas invasoras que limitaban la investigación científica. Actualmente, las tecnologías que permiten visualizar las estructuras neurológicas, como la tomografía a base de la emisión de positrones (PET), la resonancia magnética funcional (fMRI) y la tomografía computadorizada a base de la emisión de fotones aislados (SPECT), son como ventanas por las que se pueden observar los cambios que tienen lugar en el cerebro a medida que la droga entra y sale. También se pueden medir las alteraciones de la función cerebral que se producen a largo plazo cuando una persona abusa crónicamente de las drogas. Una ventaja adicional es que se puede entrevistar a un individuo que acaba de consumir una droga y correlacionar simultáneamente sus respuestas con los cambios cerebrales observados.

Por muchos años se ha sabido que cuando se expone a los drogadictos a ciertos estímulos, como cuando se les enseñan jeringas $u$ otros objetos propios del mundo de las drogas, se desencadena un deseo de consumo irresistible. Mediante las nuevas técnicas de visualización, los científicos pueden observar los circuitos cerebrales que se activan durante este proceso de estimulación. Hasta ahora se han identificado tres zonas cerebrales - la amíg- 
dala, el cerebelo y la parte lateral de la corteza prefrontal- que podrían servir de blanco para la farmacoterapia u otros tratamientos contra el abuso de drogas. Las tres zonas identificadas se asocian con la memoria y la amígdala en particular reviste los recuerdos de una aureola emocional. Esto sugiere que los aspectos emotivos de la memoria, además de los cognoscitivos, tienen importancia decisiva en la farmacodependencia. En general, las nuevas técnicas de visualización no solo arrojan luz sobre la complejidad de los procesos que intervienen en la drogadicción, sino que constituyen herramientas promisorias para observar directamente los efectos cerebrales de los tratamientos farmacológicos y conductuales empleados para curarla. (Leshner AI. NIDA's brain imaging studies serve as powerful tools to improve drug abuse treatment. NIDA Notes 1996;11:3-6; Swan N. Nida brain imaging research links cue-induced craving to structures involved in memory. NIDA Notes 1996;11:10-11).

\section{Cepas de Streptococcus pneumoniae resistentes a antibióticos en niños chilenos}

Las infecciones producidas por Streptococcus pneumoniae son las principales enfermedades bacterianas de la infancia en todo el mundo. En países en desarrollo ocasionan más de un millón de defunciones infantiles por neumonía y son causa importante de hospitalización y muerte en personas de edad avanzada.

Recientemente se han observado cambios en la epidemiología de $S$. pneumoniae, entre ellos una mayor incidencia de infecciones, algunos brotes epidémicos y la aparición y rápida extensión de la resistencia a los agentes antimicrobianos. Los estudios en portadores sirven para monitorear la resistencia a los antibióticos y la frecuencia relativa de distintos serotipos. Con el propósito de describir la prevalencia de cepas resistentes de S. pneumoniae y sus serotipos más frecuentes en niños chilenos, se tomaron muestras de las secreciones nasofaríngeas de 200 niños entre las edades de 3 meses y 4 años que asistían a guarderías infantiles en Santiago, Chile. De estos niños, 97\% tenían menos de 2 años. Las muestras se sembraron en platillos de agar con sangre de carnero, con o sin gentamicina a una concentración de $5 \mu \mathrm{g} / \mathrm{mL}$, y se incubaron a $37{ }^{\circ} \mathrm{C}$ en dióxido de carbono al 5\%. Se determinó la susceptibilidad de las cepas de S. pneumoniae a la penicilina, vancomicina, eritromicina, cefotaxima y cloranfenicol. La concentración mínima inhibidora de cada antibiótico se obtuvo mediante el uso de tiras con un gradiente exponencial de penicilina entre $32 \mathrm{y}$ $0,002 \mu \mathrm{g} / \mathrm{mL}$ (prueba E), el rastreo con disco de oxacilina y la microdilución en caldo.
Se aisló S. pneumoniae de la nasofaringe de $60,2 \%$ de los niños estudiados. De las muestras obtenidas, $28,4 \%$ mostraron resistencia a la penicilina (moderada en $20,3 \%$ de los casos y completa en $8,1 \%)$ y $2 \%$ al cloranfenicol. No se identificaron cepas resistentes a la eritromicina, la cefotaxima o la vancomicina. Los patrones de resistencia de las cepas obtenidas de niños menores y mayores de 2 años fueron similares. De los distintos serotipos, el $6 \mathrm{~A}$ fue el que se aisló con más frecuencia. El estudio, que confirmó que en Chile existe un problema de resistencia, también mostró una asociación entre la presencia de $S$. pneumoniae en secreciones nasofaríngeas y el uso reciente de antibióticos, antecedentes de enfermedad respiratoria reciente y hacinamiento familiar. En cambio, no reveló ninguna asociación con el sexo, la edad, la hospitalización reciente o la presencia de otras enfermedades. (Trucco A O, et al. Prevalencia de Streptococcus pneumoniae resistente a penicilina en niños que asisten a jardines infantiles en Santiago. Rev Chil Pediatr 1996;67:195-199).

\section{El embarazo y uso de anticonceptivos en adolescentes}

En Venezuela, país con una población predominantemente joven, los hábitos sexuales de la juventud han venido cambiando durante los últimos tres decenios. En los años sesenta, las adolescentes de 14 años de edad o menos constituían apenas $0,2 \%$ de los ingresos a servicios de obstetricia; en la década actual, esta cifra ha ascendido a $13 \%$. Según la OMS, se trata de un grupo en alto riesgo de tener embarazos no planificados. En países desarrollados, las mujeres menores de 20 años tienen más de $26 \%$ de todos los abortos, pese a que representan solo $10 \%$ de la población femenina en edad reproductiva. En 1994, la Asociación Estadounidense para el Avance de la Ciencia declaró que $81,7 \%$ de los embarazos en mujeres de 15 a 19 años eran inesperados y que $51 \%$ de ellos terminaban en un aborto.

A fin de estudiar los conocimientos, actitudes y prácticas de las adolescentes en torno al uso de los anticonceptivos, se encuestó a 100 mujeres de 14 a 19 años de edad y de estrato socioeconómico predominantemente bajo que consultaron al servicio de planificación familiar de la Maternidad Concepción Palacios de Caracas. Los resultados revelaron que $50 \%$ de las mujeres estudiadas mantenían una unión libre con su pareja sexual y que $80 \%$ habían quedado embarazadas antes de acudir al consultorio. La menarquia y la primera relación sexual habían ocurrido a una edad promedio de 12,14 y 15,04 años, respectivamente. Más de $70 \%$ de las 
adolescentes encuestadas estaban familiarizadas con algún método anticonceptivo cuando acudieron al centro de planificación familiar, pero en $90 \%$ de los casos la primera relación sexual había tenido lugar sin ningún tipo de protección contra el embarazo. Se encontró que $20 \%$ de las mujeres adolescentes habían quedado embarazadas en un plazo de 3 meses después de su primera relación sexual. Los métodos anticonceptivos mejor conocidos y más usados fueron la píldora y los dispositivos intrauterinos. (Teppa A, et al. Adolescentes: anticoncepción y experiencia sexual. Rev Obstet Ginecol Venez 1996;56:215-219).

\section{Síntomas emocionales y funciones familiares de la mujer mexicana}

En los últimos años se ha presenciado un notable aumento de los estudios sociológicos y antropológicos sobre la mujer mexicana, pero los estudios de carácter psicológico han tenido menos popularidad. Según varios estudios epidemiológicos, en México, como en otros países, los síntomas de depresión son más frecuentes en la mujer que en el hombre. Con el fin de comprender más a fondo cómo se manifiestan los problemas emocionales y cuán frecuentes son en la población femenina mexicana, se pidió a 211 mujeres que hicieran un dibujo representativo de su vida familiar. Los dibujos fueron interpretados con un enfoque tradicional y según más de una docena de categorías sintomáticas preestablecidas, como la somatización, la impulsividad, la inhibición de las emociones y la represión de hostilidad.

Según los resultados, casi $80 \%$ de las mujeres sufren de síntomas depresivos y ansiedad, $68 \%$ de ambivalencia afectiva y $99 \%$ de insatisfacción en sus relaciones personales. Muchas se ven rodeadas de un ambiente de intranquilidad y distanciamiento entre miembros de la familia. Por otra parte, la mayoría de las mujeres parecen estar bien arraigadas en la realidad y ser capaces de planificar y organizar sus numerosas tareas domésticas y laborales. Según un análisis basado en la perspectiva de género, las mujeres se desenvuelven en condiciones caracterizadas por un sistema de patriarcado y de "cautiverio" en que imperan las funciones femeninas de madre y esposa. Las malas condiciones en que se encuentra la economía mexicana impiden que las mujeres logren sus metas en el campo laboral y la sensación de frustración que ello produce es en parte el factor responsable del desequilibrio emocional. Otro factor que también contribuye al problema es el hecho de que las mujeres tienden a percibir su situación como un "fracaso" personal, sin reparar en el carácter determinante de las condicio- nes sociales a su alrededor. La represión continua de la hostilidad que eso genera, junto con la subordinación a la pareja y el acatamiento programado del ideal materno impuesto por la sociedad, llevan a la inestabilidad emocional, al autorreproche y a sentimientos de inferioridad. (Lara MA, et al. Síntomas emocionales y roles familiares en mujeres mexicanas: estudio proyectivo e interpretación de género. Acta Psiquiat Psicol Am Lat 1996;42: 329-340.)

\section{Contribución del sistema de salud cubano al bienestar de la población}

En los últimos 35 años, la situación de salud en Cuba ha cambiado favorablemente, según demuestran los indicadores de salud poblacional, que en la actualidad se asemejan a los de países más desarrollados. Hoy en día se sabe que el estado de salud de cualquier sociedad se ve determinado por el estilo de vida social e individual, el medio ambiente, la constitución genética y biológica de los habitantes y la disponibilidad de los servicios de salud. En el caso de Cuba, la salud del pueblo ha mejorado gracias a las consecuencias socioeconómicas de un proyecto social basado en los conceptos de justicia y equidad y al consiguiente mejoramiento de las condiciones de vida de los estratos pobres. Los servicios de salud han aumentado su cobertura y mejorado notablemente en organización y calidad. Iniciativas tales como el Programa de Atención Integral a la Familia, que se basa en un enfoque de riesgo, combina las actividades curativas tradicionales con las de tipo preventivo y con la promoción de la salud. A la combinación coherente y sistemática de estos dos enfoques se suma la reorientación de los servicios de salud, que recién cobra relevancia en la literatura internacional en el campo de la salud pública.

Las intervenciones de tipo poblacional atacan los problemas de salud desde la raíz y por lo tanto disminuyen su incidencia. Aunque son menos beneficiosas en términos individuales y se dirigen indistintamente a personas con diferentes niveles de riesgo, surten un gran efecto en la sociedad en general, puesto que fomentan comportamientos que a la larga mejoran el estado de salud colectivo. Por su misma naturaleza, tales intervenciones requieren la participación de varios sectores.

Las estrategias basadas en el riesgo individual son, por otro lado, fundamentalmente médicas y por lo tanto son responsabilidad del sector de la salud. Suelen gozar de aceptabilidad entre sus destinatarios y entre el personal de salud que las aplica, ya que sus beneficios son más patentes. Permiten, además, enfocar los recursos en los individuos en mayor riesgo de enfermar. Su principal 
desventaja es que suelen ser costosas, que dependen de la identificación de las personas y grupos en riesgo y que no alteran el comportamiento de las comunidades.

En Cuba, las dos estrategias antedichas se acompañan de una descentralización de los servicios de salud. La estructura provincial y municipal de las asambleas y de los consejos de salud populares facilitan el desarrollo de intervenciones comunitarias dictadas por el diagnóstico de salud y las necesidades verdaderas de cada localidad. (Espinosa Brito A. A propósito de la constitución de la Red Cubana de Municipios por la Salud. Rev Cubana Salud Publica 1995;21:113-118).

\section{Control de costos y del uso irreflexivo de las tecnologías}

El sistema de salud alemán, como el de muchos otros países, está sufriendo una transformación caracterizada por un mayor énfasis en el uso eficiente y racional de los recursos disponibles. En los años ochenta, Alemania logró mantener sus gastos de salud en una cifra estable cercana a $8,7 \%$ del producto interno bruto (PIB), sin sacrificar la cobertura universal de los servicios ni hacer cambios estructurales o alterar el modelo de financiamiento. A principios de los años noventa, sin embargo, los gastos subieron a 9,1\% del PIB y con ello surgió la necesidad de encontrar formas de racionalizar y controlar los costos.

Las medidas adoptadas como consecuencia de la situación citada se orientaron principalmente a establecer parámetros para evaluar el desempeño médico, especialmente en lo concerniente al uso de tecnologías. Hay quienes sostienen que al diseñar dichas medidas no se tuvieron en cuenta las prioridades de salud de la población alemana y que toda la energía se destinó a reducir a un mínimo los costos de la tecnología, más que a limitar su uso, en parte por temor a las posibles consecuencias legales de esto último. Aunque la fracción del PIB destinada a los gastos del sistema de salud se ha mantenido relativamente estable, el gasto por persona ha venido aumentando. No obstante, la experiencia alemana es fuente de valiosas lecciones que pueden ayudar a los países en desarrollo a idear estrategias para frenar los costos de la atención de salud.

La primera es que el sector privado puede ser un socio eficiente siempre que su participación esté regulada por directrices políticas serias y claras. La segunda es que se pueden adoptar medidas explícitas para el control de costos, aunque no sean populares entre los médicos, si se apoyan en datos contundentes y objetivos obtenidos por medio de un sistema de información confiable y tras un proceso de negociación abierto entre las partes. En tal caso se torna imprescindible establecer parámetros bien definidos, ajustados a la realidad nacional, para evaluar el uso y desempeño de las numerosas tecnologías modernas que automática e irreflexivamente se incorporan al sistema de salud en la mayor parte de los países del mundo en desarrollo. La tercera lección, que es la más importante, consiste en reconocer la presencia de un sesgo según el cual el uso de tecnologías complejas se toma como falso indicador de un buen rendimiento institucional o profesional. Las medidas adoptadas en Alemania tuvieron por objeto disminuir "el consumo imperativo de las tecnologías médicas". Al fin y al cabo, aún no se ha comprobado que dichas tecnologías, pese a su capacidad de prolongar la vida de los individuos, mejoren la esperanza de vida de la población en general. (Pitelli da Guia RG. Controlando a utilizaçao indiscriminada de tecnologias médicas e a escalada dos custos dos sistemas de saúde: a estratégia alemã. Cad Saude Publica (Rio de Janeiro) 1996;12:159-170).

\section{¿Existen alimentos que protejan contra los trastornos coronarios?}

Los procesos ateroscleróticos, y especialmente la cardiopatía coronaria, guardan relación con varios factores de riesgo que aumentan las concentraciones séricas de colesterol, como la obesidad y la falta de ejercicio. De 1 a $2 \%$ de la población estadounidense tiene hipercolesterolemia de origen genético, pero en países desarrollados la elevación del colesterol en sangre se debe casi invariablemente a una alimentación inadecuada. Varios estudios epidemiológicos han demostrado que la cardiopatía coronaria y la trombosis son menos frecuentes en poblaciones cuyo régimen alimentario es bajo en grasas y en colesterol.

Es un hecho comprobado que las concentraciones del colesterol ligado a lipoproteínas de baja densidad (colesterol-LDL) se asocian con el riesgo de cardiopatía coronaria. Las grasas saturadas elevan las concentraciones totales de colesterol. El consiguiente bloqueo de la actividad de los receptores de LDL, que dificulta la eliminación de estos lípidos, hace que suba la concentración de colestrolLDL. Los ácidos grasos que más elevan las concentraciones de este tipo de colesterol son el ácido palmítico y el ácido mirístico; en cambio, los ácidos esteárico y oleico producen un aumento menos marcado. A ello se atribuye que la población de España y de otros países donde se consume mucha carne de cerdo, que es rica en ácido esteárico, ten- 
gan concentraciones de colesterol menores de lo que cabría esperar. Sucede lo contrario en poblaciones que consumen mucha mantequilla, por ser esta más rica en ácidos mirístico y palmítico. El ácido láurico también produce hipercolesterolemia, pero en menor medida. Ni los ácidos grasos de cadena mediana ni los carbohidratos o las grasas monoinsaturadas elevan el colesterol sanguíneo y deben reemplazar a otros ácidos grasos más peligrosos.

Las partículas de HDL facilitan el transporte de colesterol hasta el hígado, donde se metaboliza. Por lo tanto el colesterol ligado a lipoproteínas de alta densidad (colesterol-HDL) reduce el riesgo de cardiopatía coronaria. La razón de colesterol-LDL a colesterol-HDL es un indicador fiable del riesgo de padecer trastornos de las coronarias. En poblaciones donde los individuos tienen altas concentraciones de ambos tipos de colesterol, las personas en quienes esta razón es alta están en mayor riesgo de cardiopatía coronaria. Aunque los genes ejercen su influencia en esta relación, la dieta es un factor determinante. El consumo de ácidos grasos saturados es dañino porque produce un aumento de la concentración de colesterol-LDL. (Gutiérrez Fuentes JA. ¿Qué alimentos convienen al corazón? Foro Mundial Salud 1996;17:164-171).

\section{Director General de la OMS repudia la clonación humana}

Al enterarse del éxito obtenido por un equipo de científicos escoceses que lograron clonar una oveja adulta, el doctor Hiroshi Nakajima, Director General de la Organización Mundial de la Salud, hizo una declaración pública en la que repudió la idea de clonar a los humanos: "La OMS considera inaceptable desde el punto de vista ético el uso de la clonación como forma de duplicar a un ser humano; ello violaría algunos de los principios básicos que rigen la procreación por intervención médica. Entre estos se incluyen respetar la dignidad del ser humano y proteger el material genético humano".

En 1992 un grupo científico convocado por la OMS para examinar los aspectos técnicos y éticos de la procreación asistida defendió el derecho de beneficiarse de las aplicaciones del progreso científico. Defendió además la libertad indispensable para poder investigar creativamente. No obstante, el grupo hizo hincapié en el consenso universal de prohibir formas extremas de experimentación, como la clonación humana, la fecundación entre especies distintas, la creación de quimeras y la alteración del genoma de las células reproductoras.

El doctor Nakajima dejó claramente establecido que la OMS no se opone a los procedimientos ordinarios de clonación para producir anticuerpos monoclonales y otros productos para diagnóstico y tratamiento de enfermedades. La OMS, por lo tanto, ha empezado a encabezar los debates internacionales que se requieren para establecer buenas prácticas, directrices y legislación en esta materia. 